\title{
Primary Hypertension in Children and Adolescents is an Immuno-Metabolic Disease with Hemodynamic Consequences
}

\author{
Mieczysław Litwin • Jacek Michałkiewicz • \\ Lidia Gackowska
}

Published online: 5 June 2013

(C) The Author(s) 2013. This article is published with open access at Springerlink.com

\begin{abstract}
With the rise in obesity epidemic primary hypertension $(\mathrm{PH})$ is now one of the most common chronic diseases in adolescence. In contrast to hypertensive adults, hypertensive children usually are not exposed to other comorbidities such as diabetes, chronic kidney disease and atherosclerosis. Thus, PH in children and adolescents can be treated as the early stage of development of cardiovascular disease. There is increasing amount of data indicating that $\mathrm{PH}$ is not only hemodynamic phenomenon but a complex syndrome involving disturbed activity of sympathetic nervous system, metabolic abnormalities and activation of innate and adaptive immune system. We discuss results of the studies on clinical, metabolic and immunological phenotype of hypertensive children, associations between metabolic and immunological abnormalities with target organ damage and results of antihypertensive treatment.
\end{abstract}

Keywords Primary hypertension $\cdot \mathrm{PH} \cdot$ Children . Adolescents · Immune activity $\cdot$ Cytokines $\cdot$ T regulatory cells $\cdot$ Metabolic syndrome $\cdot$ Visceral obesity $\cdot$ Blood pressure $\cdot$ BP $\cdot$ Cardiovascular disease $\cdot$ CVD $\cdot$ Target organ damage $\cdot$ TOD $\cdot$ Treatment

M. Litwin $(\triangle)$

Department of Nephrology and Arterial Hypertension, The Children's Memorial Health Institute, Warsaw, Poland e-mail: m.litwin@czd.pl

J. Michałkiewicz

Department of Microbiology and Immunology, The Children's

Memorial Health Institute, Warsaw, Poland

J. Michałkiewicz $\cdot$ L. Gackowska

Chair of Immunology, Collegium Medicum, Bydgoszcz, Nicolaus

Copernicus University, Torun, Poland

\section{Introduction}

Cardiovascular disease (CVD) has its origin in childhood and may be programmed perinatally [1]. One of the main risk factors of CVD is primary hypertension $(\mathrm{PH})$. With the rise in obesity epidemic $\mathrm{PH}$ has become one of the most common childhood diseases. The frequency of arterial hypertension in $8-18$ years-old children is about $2-3.6 \%$ and is almost $10 \%$ among 18 years old adolescents [2]. Although clinically evident complications of $\mathrm{PH}$ do not appear before the fourth to fifth decade of life, hypertensive target organ damage (TOD) is evident already in hypertensive children [3]. There is increasing amount of data indicating that PH is not only a hemodynamic phenomenon, but a complex disease involving sympathetic nervous system, metabolism and immune system. Below, we discuss results of the studies on clinical, metabolic and immunological phenotype of hypertensive children, associations between metabolic and immunological abnormalities with TOD and results of antihypertensive treatment. Finally, hypotheses linking immune and metabolic abnormalities with elevated blood pressure are presented.

\section{Clinical Phenotype of PH in Childhood}

Blood pressure (BP) values in children strictly correlate with biological development and body size. Therefore, assuming that height and age are the main determinants of BP, referential BP values have been adjusted according to age and height. However, obesity epidemic changed this relationship. A population study on almost 18,000 randomly chosen, healthy school age children clearly showed that body mass but not height determined population BP. Moreover, it 
was waist circumference (WC), increased body mass and lower height what were the main predictors of BP [4]. Relations between anthropometrical parameters and BP are even more evident in children with PH. The typical intermediary phenotype, overweight, obesity and increased body mass index (BMI) may serve as the discriminating factors in differential diagnosis of $\mathrm{PH}$ in adolescents [4-8]. More than $20 \%$ of children with PH in the USA and Europe are obese or overweight $[4,9]$ and analysis of body composition showed that children with $\mathrm{PH}$ had higher fat mass and low lean body mass/fat mass ratio in comparison with normotensive children [7].

There are data indicating that maintenance of normal BMI and body composition protects against BP rise. The normotensive or prehypertensive adults in the mean age of 60 years who did not increase BMI and/or decreased BMI or WC during the 5-year follow-up period, showed no increase in their BP. Similarly, the fit subjects more often presented normal blood pressure than the non-fit subjects [10••]. Similarly, the young men who increased theirs BMI during the 10 years period had greater BP than those who maintained normal weight [11].

\section{Accelerated Rate of Biological Development}

Based on the observations of Pickering's group, Lever and Harrap in 1992 proposed that PH is a disorder of growth with origins in childhood $[12 \bullet \bullet, 13]$. According to it, hypertrophy of arterial wall smooth muscle cells and metabolic changes, including insulin resistance (IR) may be at least in part associated with accelerated biological maturation, which together may lead to hypertension and CVD.

In 1980 Katz et al, described accelerated biological development expressed as bone age in hypertensive children [14•]. In the study of hypertensive boys it was found that rate of biological development expressed as the difference between bone age and chronological age, was increased by 1.5 years in comparison with age and BMI matched peers [15•]. In addition, there was linear relationship between acceleration of bone growth with BP status from normotension, through prehypertension, stage 1 to stage 2 hypertension. Growth spurt, another marker of maturity (in Poland about 13.5 year of age), was found to be strictly related with a significant rise in BP values in boys but not in girls [16, 17•]. It fits well with observation that the ratio of boys to girls among hypertensive adolescents is $2-3$ : 1 . These findings were confirmed in retrospective study from Iceland. It was shown that adult males who had the highest growth velocity between age 8 and 13, had $66 \%$ increased risk of hypertension in comparison with males who had lowest growth velocity [17•]. Similarly, in the prospective Young Finns Study woman who had been younger at menarche were hypertensive, had metabolic syndrome (MS) and were viscerally obese in fourth decade of life $[18 \bullet]$.

\section{Biochemical Phenotype of Hypertensive Children}

Childhood $\mathrm{PH}$ is characterized by biochemical phenotype typical for metabolic abnormalities associated with visceral obesity, such as hypertriglycerydemia, low HDL-cholesterol, glucose intolerance and IR. It is estimated that prevalence of MS equals 15 to $20 \%$ among adolescents with $\mathrm{PH}$ and is 10 times more prevalent than in general adolescent population [19].

The other typical metabolic abnormality found in children with $\mathrm{PH}$ is tendency to greater concentrations of uric acid. It was found that $89 \%$ of children with PH had serum uric acid concentrations above $5.5 \mathrm{mg} / \mathrm{dl}$ in comparison with $30 \%$ of children with secondary hypertension and none with white coat hypertension and normotensive controls [20•]. Moreover, treatment with allopurinol or probenecid caused not only decrease of serum uric acid concentrations but also was associated with blood pressure decrease in obese, stage 1 hypertensive and prehypertensive children $[21,22 \bullet]$.

Increased oxidative stress (SOX) has been described in adults with $\mathrm{PH}$, but there are only few data from pediatric studies on SOX in PH [23, 24•]. It was found that the hypertensive children had decreased levels of antioxidative defense system and elevated concentrations of reactive oxygen species (ROS). In the prospective study of $86 \mathrm{PH}$ children, those with severe ambulatory hypertension had lower concentrations of glutathion than children with ambulatory hypertension [24•]. Furthermore, hypertensive children with MS had lower activity of glutathione peroxidase, higher serum concentrations of asymmetric dimethylarginine (ADMA) and oxidized LDL-cholesterol (oxyLDL) than hypertensive children without MS. SOX was also associated with left ventricular hypertrophy.

\section{Immunological Characteristics of Hypertensive Children}

There is a significant graded relationship between BP and serum sICAM-1 and IL-6 concentrations indicating that increased BP is correlated and/or may stimulate systemic lowgrade inflammation [25]. Chronic subclinical inflammation is considered as a part of the MS [26]. Moreover, inflammatory markers predict the development of diabetes and of IR [27]. The sub-acute inflammation leading to vascular inflammation and hypertension can be activated by: (1) ongoing intraarterial inflammation mediated by wall-resident macrophages which respond to various endogenous inducers, (2) low-level extravascular stimuli as smoking, aging, obesity, mucosal or 
oral infections such as bronchiltis, gastritis, or periodontitis, and (c) the hemodynamic insult caused by elevated blood pressure and pulse pressure. The resulting inflammatory responses are associated with IR and play role in pathogenesis of CVD [28].

Analysis of the immune profile of hypertensive adults was estimated on the basis of serum level concentration of many inflammatory mediators such as acute phase proteins (like CRP), cytokines, chemokines, metalloproteinases (MMP), collagen degradation products, oxidative stress markers, adipokines, including leptin, adiponectin, resistin, etc. The results confirmed that $\mathrm{PH}$ is associated with ongoing systemic inflammation. This approach however has some limitations. Most inflammatory mediators act as autocrine/paracrine factors, so their circulating levels may not reflect their pathophysiological roles. It is also difficult to indicate if changes in their serum levels are primary or secondary to the ongoing inflammatory process. This concerns especially the adult hypertensive patients who are often exposed to many confounders such as smoking, drugs, diet customs, etc. In study including only children with $\mathrm{PH}$ we found that hypertensive children had greater serum concentrations of highly sensitive $\mathrm{C}$ reactive protein (hsCRP) and chemokines (RANTES, MIP1 $\beta$ ) in comparison with normotensive children. hsCRP levels correlated with MS, visceral obesity, SOX and markers of TOD such as increased carotid intima-media thickness (cIMT) and left ventricular mass. RANTES levels correlated with dyslipidemia. Elevated hsCRP levels did not correlate neither with RANTES nor MIP1 $\beta$. RANTES acts mainly as a memory $\mathrm{T}$ cell attractant, but MIP-1 $\beta$ is responsible for monocyte attraction to the inflammation site. Therefore lack of correlation between their levels and systemic inflammation marker such as hsCRP may suggest that vascular inflammation somehow precedes systemic inflammatory events [29]. These results indicate that systemic immune activation already occurs in the early stage of $\mathrm{PH}$.

Activation of both innate and adaptive immune system has been shown to play role in pathogenesis of $\mathrm{PH}$ in experimental models and in humans [rev. in 30, 31••]. Cells of the immune system, including peripheral blood leucocytes (PBL) contain full enzymatic machinery of renin-angiotensin system (RAS), the central player in circulatory homeostasis [32, 33]. Moreover, angiotensin 2 (AT2) and angiotensin receptor blockers (ARB) modulate reciprocally function of both innate and adaptive immune system [33, 34]. Chon et al. analyzed genes expression in leukocytes of six untreated and six treated hypertensive adults and found that in untreated patients expression of 680 genes was altered, of which 314 were upregulated and 366 were dow-regulated. On the contrary, in treated patients, who had significantly lower BP, only four genes were up-regulated and three were down-regulated. Among upregulated genes were those involved in inflammatory reaction, antioxidative defense and RAS. Furthermore, normalization of
BP corrected these changes [35]. Coppo et al. found in PBL from hypertensive adults upregulated expression of 21 genes known to be engaged in apoptosis, inflammatory responses, vesicular trafficking of molecules among cellular organelles, and other processes [36]. In yet another study, the expression pattern of the majority of genes was similar regardless the disease stage and antihypertensive therapy [37]. In pediatric study, PBL from untreated children with $\mathrm{PH}$ had increased expression of mRNA of ACE and CD14. Angiotensinogen (AGT) and AT2 type 1 receptor (AT2R1) mRNAs expression were down regulated. Moreover, children with increased cIMT had significantly lower renin (REN) mRNA expression [38]. The reason for that is not clear, but strongly suggests that RAS system in PBL must be activated early in the course of PH.

These reports confirm and extend the idea that $\mathrm{PH}$ is associated with complex inflammatory responses which engage many genes involved both in immune system activation and BP regulation. The changes in their expression can be found in PBL so it makes possible to eventually classify the disease, its clinical course and responsiveness to treatment. These studies also indicate that leukocytes may serve as "sensors" of PH. However, results of above mentioned studies do not indicate which cells of immune system initiate the inflammatory cascade resulting in hypertension. It is known from experimental studies with genetically modified mouse, that hypertension is associated with $\mathrm{T}$ cells and macrophages. According to hypothesis proposed by Harrison et al. these cells infiltrate kidney and vasculature which are crucial for BP control $[31 \bullet \cdot]$. According to this hypothesis, modest BP elevation results in activation of endothelium. Activated endothelium reveals neoantigens which are taken up by dendritic cells and present them to T cells recognizing them as non-self in the regional lymph nodes. Non-self recognition induces strong $\mathrm{T}$ cells activation, proliferation and differentiation. In the prehypertension stage, the pool of sensitized $\mathrm{T}$ cells is relatively small but their numbers increase with subject age so does inflammation. The most important subset of $\mathrm{T}$ cells engaged in inflammatory reaction and elevation of BP is Th17 T cell subset. Th17 produce IL-17 and are engaged in many autoimmune diseases including rheumatoid arthritis, inflammatory bowel disease, psoriasis, sclerosis multiplex, SLE, etc. IL-17 exerts its effect by production of chemokines and adhesion molecules in tissue. This leads to accumulation of other inflammatory cells in the vascular wall (kidney, peripheral vessel wall), promotes ROS production in the vascular smooth muscle cells and decreases nitric oxide synthesis by endothelial cells. It causes vasoconstriction, sodium retention and hypertension [39]. It was found that mice lacking Tlymphocytes and B lymphocytes are resistant to development of both angiotensin II, DOCA-salt, epinephrine, or stressinduced hypertension. On the contrary, adoptive transfer of $\mathrm{T}$ cells, but not of B lymphocytes, restored hypertensive response to these stimuli $[40 \bullet \bullet, 41]$. 
Inflammation is controlled by T-regulatory cells (T-regs) which limit the extent of the response and prevents the tissue damage (in this case arteriolar wall causing hypertension). T-regs can stop inflammation by acting on T cells, B cells, macrophages, dendritic cells and NK lymphocytes. In the mouse model, adoptive transfer of T-regs limited hypertension and organ damage induced either by AT2 or aldosteron $[42 \bullet \bullet]$. These results indicate that T-regs can be generated in the course of hypertension at least in the experimental models with use of stimuli such as high salt feeding, AT2, and aldosterone. In our yet unpublished study of untreated children with $\mathrm{PH}$, we found that PBL from hypertensive children responded more vigorously (as assessed by cytokines expression profiles) to $\mathrm{T}$ cell stimuli such as lipopolysacharide, than PBL from normotensive children with non-alcoholic fatty liver disease and from healthy children. In addition, CD4 from children with PH had increased T-regs and Th17 cell population in comparison with normotensive, age and BMI matched children. Additionally, the number of Th17 cells was greater in those patients whose leukocytes did not express adiponectin type 1 receptors (AdipoR1) (unpublished results). This indicates that decreased AdipoR1 expression may limit leukocyte responsiveness to adiponectin. This in turn may increase synthesis of proinflammatory IL-17 by T cells (Fig. 1). These observations are an argument for significance of adipose tissue and adipokines in the regulation of the inflammatory responses in hypertensive children.

Signals which lead to differentiation of naive CD4+ T cells into proinflammatory Th17 cells require IL-6 and TGF-beta. In contrast, T-regs need TGF-beta-an antiinflammatory cytokine produced mainly by macrophages and monocytes - for their differentiation from naïve CD4+ T cells and synthesis of FOXP-3 by T-regs. In contrast, IL6-a ubiquitous and multifunctional cytokine which can be produced by many cell types, including $\mathrm{T}$ and $\mathrm{B}$ cells, fibroblasts, endothelial cells, macrophages, adipose tissue macrophages, monocytes, and dendritic cells - is a high inducer of acute phase response and its serum concentrations correlate with BP in healthy subjects.

There is strictly controlled relation between T-regs and Th17 cells. In the presence of TGF-beta but of low IL-6 concentrations, naive $\mathrm{T}$ cells preferentially polarize into $\mathrm{T}$ regs, and Th17 cells may transit into T-regs. However, when cytokines milieu contains mainly pro-inflammatory cytokines such as IL-6 and low TGF-beta concentrations, then T cells preferentially polarize into Th17, and T-regs may lose FOXP3 expression and transit into Th17 cells. It suggests that type of inflammatory reactions generated during initial phases of responses to vascular antigens may decide about the immune response profile. According to this scenario it was described recently that IL- 6 accumulates in the kidney, especially the glomeruli, of patients with chronic kidney disease and hypertension, to a greater extent than in normotensive patients with CKD [43].

RAS in immune cells may be an autonomous source of AT2 independently of circulating and tissue RAS [44]. Proinflammatory signals may increase immune system cells sensitivity to AT2. For example, T cells of hypertensive adult patients showed no changes in baseline mRNAs expression for angiotensin converting enzyme (ACE) and AT2R1 but stimulation with angiotensin II resulted in rapid increase in both ACE and AT2R1 expression. Systemic inflammation expressed as increase in serum CRP level, correlated with $\mathrm{T}$ cells response to AT2, indicating that AT2-driven T cell RAS was further up-regulated by lowgrade inflammation [36].

The consequence of immune activation and hemodynamic injury is extracellular remodeling and fibrosis. The main controlling system of extracellular matrix remodeling is system of matrix metalloproteinases (MMP) and its tissue inhibitors (TIMP). The results of recently published metaanalysis indicate that both concentrations and activities of MMP-9 and TIMP-1 are disturbed in adults with hypertension and correlate with left ventricular hypertrophy [45 •]. It was also found that serum adiponectin concentrations correlate negatively with MMP-9 and TIMP-1, and MMP9/TIMP-1 ratio is a predictor of stability of coronary atherosclerotic plaque and extent of coronary atherosclerosis [46]. Pediatric reports refer to children with obesity, diabetes or MS [47]. It was found that obese and hypertensive children had greater concentrations of MMP-9 than obese, normotensive children. Moreover, both MMP-9 and TIMP-1 correlated with BMI, systolic blood pressure and IR. In our study we found that hypertensive children have greater concentrations of MMP-9 in PBL and in serum in comparison with age and BMI matched normotensive children [48]. Moreover, hypertensive children with MS had greater concentrations of TIMP1 in comparison with hypertensive children without MS.

\section{Target Organ Damage in Children with Primary Hypertension}

Both left ventricular mass index (LVMI), carotid intimamedia thickness (cIMT) and arterial stiffness increase with change of BP status from normotension, through prehypertension to hypertension and correlate with severity of hypertension [49, 50]. The same was observed in children with normotension, white coat hypertension, and hypertension [51]. However, change of BP status was also accompanied by increasing adiposity and metabolic abnormalities. In studies of children with PH it was repeatedly shown that already at diagnosis left ventricular hypertrophy (LVH) expressed as LVMI above 95th percentile for age and sex, 
Adiponectin receptor expression AdipoR1 positive cells $61,47 \%$

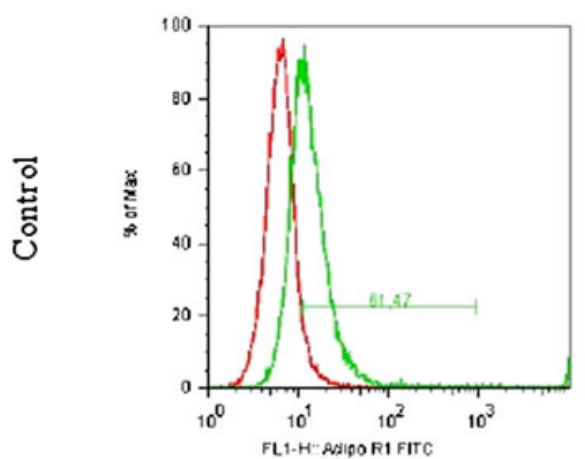

Adiponectin receptor expression

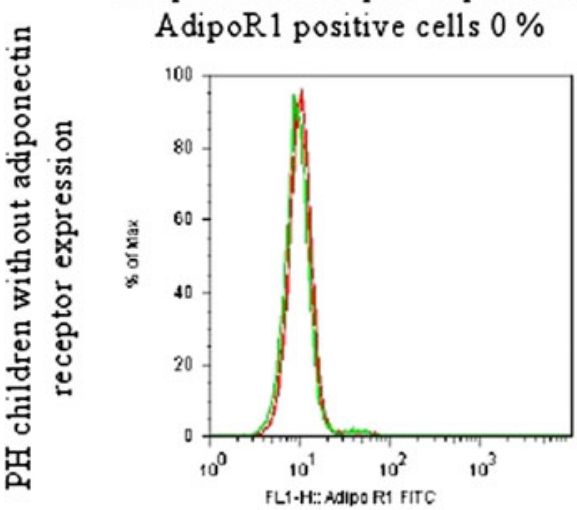

Adiponectin receptor expression AdipoR 1 positive $94,84 \%$

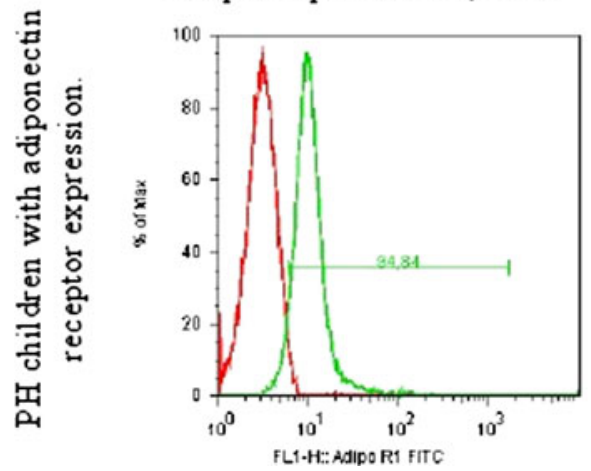

Th17 cells-CD4 $4^{+} / \mathrm{IL}-17^{+}$

(positive cells 2,07\%)

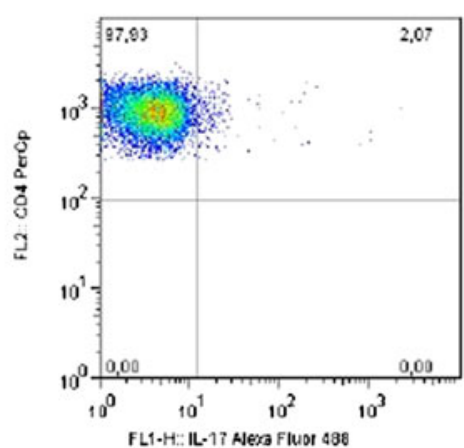

Th 17 cells-CD $4^{+} / \mathrm{IL}-17^{+}$ (positive cells 7,59\%)

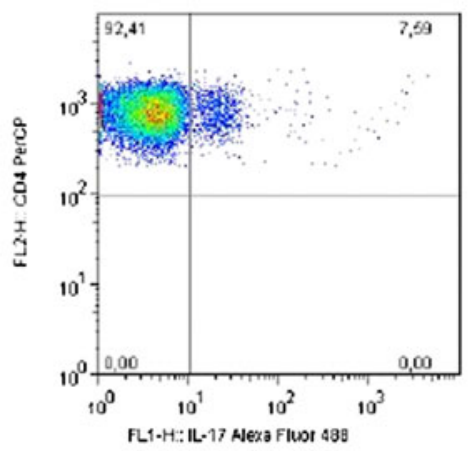

Th17 cells-CD $4^{+} / \mathrm{IL}-17^{+}$ (positive cells 2,10\%)

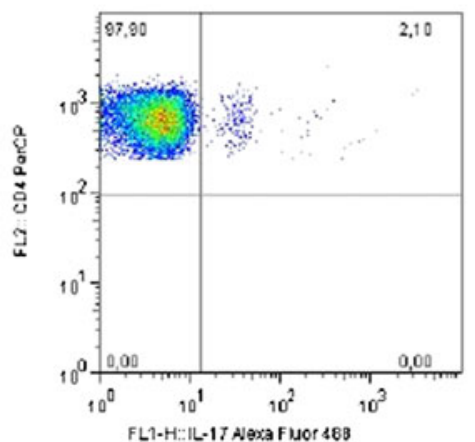

Treg $\mathrm{CD}^{+} / \mathrm{CD} 25^{\text {high }} / \mathrm{CD} 127^{\text {low }}$

(positive cells $5,41 \%$ )

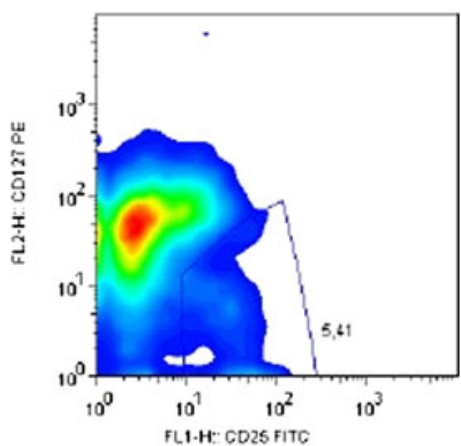

Treg $\mathrm{CD}^{+} / \mathrm{CD} 25^{\text {high }} / \mathrm{CD} 127^{\text {low }}$
(positive cells $9,26 \%$ )

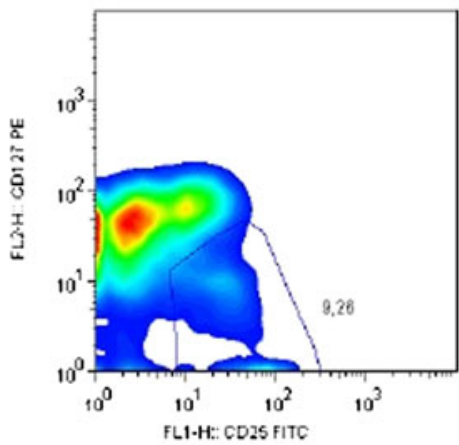

Treg $\mathrm{CD} 4^{+} / \mathrm{CD} 25^{\text {high }} / \mathrm{CD} 127^{\text {low }}$ (positive cells $8,64 \%$ )

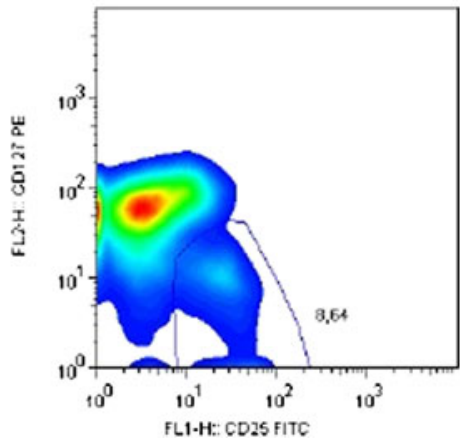

Fig. 1 Relationship between adiponectin receptor type 1 (AdipoR1) expression levels in neutrophils and numbers of IL-17 expressing CD4 $\mathrm{T}$ cells $(\mathrm{Th} 17+)$ and $\mathrm{CD} 4+\mathrm{T}$ regs cells bearing $\mathrm{CD}^{+} / \mathrm{CD} 25^{\text {high/ }}$ $\mathrm{CD} 127^{\text {low }}$ phenotype. Flow cytometry analysis of peripheral blood (PB) cells gated as neutrophils (AdipoR1 expression, left column) and CD4+ T cells bearing Th17 or T-regs phenotype (middle and right column, respectively). The plots are representatives for 25 controls and 23 PH children. Results: 1) low numbers of Th17 and T-regs in control

was present in $38-46 \%$, and severe LVH expressed as LVMI above $52 \mathrm{~g} / \mathrm{m}$ height $^{2.7}$ was found in $13-15 \%[3$, 52, 53•]. Prevalence of LVH was greater in children with positive for neutrophil AdiopoR1 expression, 2) high expression of IL17 and T-regs cells in PH children negative for neutrophil AdipoR1 expression, 3) low IL-17 expression (like in the control) in PH children bearing neutrophil AdipoR1, 4) increased T-regs numbers in PH children as compared to controls. Abbreviations: AdipoR1 - adiponectin receptor type 1; IL-17 - interleukin 17; $\mathrm{PH}$ - primary hypertension; Tregs - T regulatory cells

severe ambulatory hypertension in comparison with children who had ambulatory hypertension, and the most prevalent form of LVH in hypertensive children was eccentric 
hypertrophy [50, 52, 53•, 54, 55]. Extent and severity of TOD correlated with visceral obesity and metabolic abnormalities and it was found that prevalence of LVH increased with exposure to increasing number of MS criteria [19]. Moreover, severe LVH was found only among patients who had MS. Similarly, about $40 \%$ of children with PH had signs of hypertensive arteriopathy expressed as increased cIMT $[9,53 \bullet]$. Metabolic abnormalities, especially atherogenic dyslipidemia, aggravate arterial wall injury caused by elevated BP. In analysis of group of children with $\mathrm{PH}$, increased cIMT was predicted by pulse pressure, homocysteine concentrations, low HDL cholesterol levels, adiponectin, hsCRP, angiogenin and WC $[9,19,29,52$, 53•]. Similarly, concentrations of thiobarbituric acid substances - a marker of SOX - correlated with LVMI, concentric LVH, cIMT and albuminuria [24•].

\section{Effects of Treatment of Primary Hypertension in Children and Adolescents}

According to recent guidelines, treatment of $\mathrm{PH}$ in children and adolescents should start with nonpharmacological treatment and include both dietary changes and increased physical activity [56, 57•]. Pharmacological treatment should be added for patients with stage 2 hypertension and/or TOD. Due to typical intermediate phenotype and associated metabolic abnormalities with special emphasis on IR, the best pharmacological treatment option is use of angiotensin converting enzyme inhibitors (ACEi) or ARB. Although results of clinical trials documented that both ACEi and ARBs are effective and safe as antihypertensive drugs in children, these studies included patients mainly with secondary forms of hypertension $[58,59]$.

There are only few prospective studies evaluating effects of treatment specifically in children with PH. In our reports we analyzed results of both non-pharmacological and pharmacological treatment based on ACEi or ARB in children with $\mathrm{PH}[24 \bullet, 53 \bullet]$. The principles of treatment were as follows: pharmacological treatment was started in pts who had significant TOD and/or severe ambulatory hypertension. If after 3 or 6 months office BP was still in the hypertensive range, ambulatory blood pressure monitoring (ABPM) was done. In pts who still had ambulatory or severe ambulatory hypertension, pharmacotherapy was started or another drug was given. Drug therapy was based on an ACEi (enalapril $0.2-0.3 \mathrm{mg} / \mathrm{kg} /$ day bid), and in patients who had asthma or did not tolerate ACEi, losartan (0.$1 \mathrm{mg} / \mathrm{kg} /$ day in one or two daily doses) was prescribed. Amlodipine was used as second drug. After one year normotension has been achieved in $70 \%$ of pts, including $60 \%$ in optimal BP range and $10 \%$ in prehypertensive range. It was accompanied by significant regression of TOD expressed as LVH and increased cIMT, decrease of MS prevalence by $50 \%$ and decrease of inflammatory activity. However, concentric LVH was less prone to decrease. Moreover, despite significant lowering of BP the main predictor of TOD regression was not decrease of BP but decrease of visceral obesity expressed as decrease of WC and decrease of IR [53•, 54]. Treatment caused also significant decrease of SOX (23). Another prospective study of effects of 6 months of non-pharmacological treatment in 23 adolescents with stage $1 \mathrm{PH}$, revealed significant changes in genes expression in peripheral blood leucocytes. There was significant decrease of expression of genes of AT2R1, AGT and ACE. CD40 molecule expression decreased but nonsignificantly [38].

Observation of BP rhythms in ABPM before and after one year of antihypertensive treatment in 50 adolescent boys with $\mathrm{PH}$ gave interesting results. After 1 year of treatment, $68 \%$ of patients were normotensive, and left ventricular mass and cIMT decreased in $60 \%$ and $62 \%$ of patients respectively. However, on average, BP and heart rate patterns did not change. More detailed analysis revealed that changes in BP amplitude correlated with the decrease of WC $(p=0.035)$. Moreover, the decrease of visceral fat amount assessed by magnetic resonance, correlated with the decrease of 24-h mean arterial pressure and heart rate acrophases (both $p<0.05$ ). There were no differences in changes of $\mathrm{BP} /$ heart rate rhythms between patients who achieved or did not achieve normotension and/or regression of LVMi and cIMT [60]. Persistence of abnormal cardiovascular rhythmicity in children with $\mathrm{PH}$ despite effective antihypertensive treatment suggests that it may be the primary abnormality. However, the correlation between changes in cardiovascular rhythmicity and visceral obesity may indicate that the visceral fat plays an important role in the sympathetic activity of hypertensive adolescents.

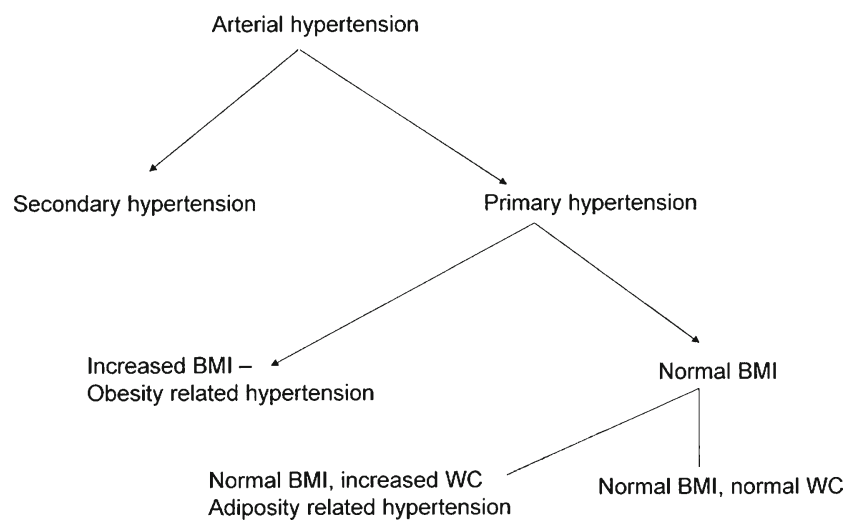

Fig. 2 Schematic classification of arterial hypertension in children and adolescents. Abbreviations: BMI - body mass index; WC - waist circumference 


\section{Conclusions}

Described above clinical, immuno-metabolic and hemodynamic phenotype of children with $\mathrm{PH}$ and determinants of treatment efficacy are in line with paradigm describing $\mathrm{PH}$ in children and adolescents as immuno-metabolic disease strictly linked to visceral obesity and disturbed body composition. There is significant relationship both between body growth, body composition, fat tissue distribution, immune and metabolic abnormalities with $\mathrm{PH}$, disturbed cardiovascular rhythms and hypertensive TOD. According to this, we propose classification of childhood hypertension as depicted on Fig. 2. In this scheme, PH can be further divided on the basis of not only of fat amount but also of fat tissue distribution. Thus $\mathrm{PH}$ in children can be divided into $\mathrm{PH}$ in children with elevated and normal BMI but with increased visceral adiposity. PH in children and adolescents with normal body composition and without metabolic abnormalities seems to be distinct entity, including cases of so known spurious hypertension [61•] Accordingly, basing on findings both from crosssectional and prospective studies, we hypothesize that it is normalization of immuno-metabolic abnormalities and body composition what determines normalization of both TOD and cardiovascular rhythmicity. Such a view considers elevation of BP in childhood as the first step in development of CVD. Usually typical clinical phenotype is accompanied by subtle immuno-metabolic abnormalities. In this stage of disease both metabolic and hemodynamic abnormalities and TOD are usually reversible and regression of TOD correlates with normalization of immune activity. However, some forms of $\mathrm{LVH}$ (concentric hypertrophy) are less prone to treatment. It is assumed that the next stage of disease, clinically evident in adulthood evolves into the third stage of irreversible cardiac, renal and vascular changes [31••]. The evolution of hypertensive disease is accompanied by evolving pattern of sodium sensitivity from renin-dependent hypertension to sodium dependent hypertension [62••].

There are still unresolved problems. There are no data linking pathogenically immune and metabolic abnormalities with disturbed cardiovascular rhythms. However, there are some hypotheses linking central autonomic activity with immune phenomena in hypertensive animals [63•]. The primacy of increased central autonomic activity or secondary to visceral adiposity and metabolic abnormalities is also unresolved. Secondly, there are no data if normalization of BP, body composition and immuno-metabolic abnormalities allows to discontinue antihypertensive treatment. It seems that even if it is possible, those subjects could be prone to develop hypertensive disease later in life. Increasing amount of data indicate that both primary prevention and antihypertensive treatment of children and adolescents with $\mathrm{PH}$ should be aimed to immunemetabolic abnormalities and disturbed body composition and not only to blood pressure lowering. However, this hypothesis should be proven in multicenter prospective trials, examining effects of different drug classes not only in terms of decrease of blood pressure but also analyzing regression of target organ damage and metabolic abnormalities.

Acknowledgments This study was supported by grants from the Polish Scientific Committee (855/B/P01/2008/34, 4552/B/P01/2009/ 37, 5412/B/P01/2010/39) and National Science Center (2011/01/B/ NZ6/02661).

Conflict of Interest Mieczysław Litwin declare that he have no conflict of interest.

Jacek Michałkiewicz declare that he have no conflict of interest.

Lidia Gackowska declare that she have no conflict of interest.

Open Access This article is distributed under the terms of the Creative Commons Attribution License which permits any use, distribution, and reproduction in any medium, provided the original author(s) and the source are credited.

\section{References}

Papers of particular interest are highlighted as:

- Of importance

- Of major importance

1. Barker DJP, Osmond C, Golding J, Kuh D, Wadsworth MEJ. Growth in utero, blood pressure in childhood, and adult life, and mortality from cardiovascular disease. BMJ. 1989;298:564-7.

2. Hansen ML, Gunn PW, Kaelber DC. Underdiagnosis of hypertension in children and adolescents. JAMA. 2007;298:874-9.

3. Daniels SR, Loggie JM, Khoury P, Kimball TR. Left ventricular geometry and severe left ventricular hypertrophy in children and adolescents with essential hypertension. Circulation. 1998;97:1907-11.

4. Kułaga Z, Litwin M, Grajda A, et al. Oscilllometric blood pressure percentiles for Polish normal-weight children. J Hypertens. 2012;30:1942-54.

5. Flynn JT, Alderman MH. Characteristics of children with primary hypertension seen at a referral center. Pediatr Nephrol. 2005;20:961-6.

6. Robinson RF, Batisky DL, Hayes JR, Nahata MC, Mahan JD. Body mass index in primary and secondary pediatric hypertension. Pediatr Nephrol. 2004;19:1379-84.

7. Pludowski P, Litwin M, Sladowska J, et al. Bone mass and body composition in children and adolescents with primary hypertension. Hypertension. 2008;51:77-83.

8. Litwin M, Śladowska J, Syczewska M, et al. Different BMI cardiovascular risk thresholds as markers of organ damage and metabolic syndrome in primary hypertension. Pediatr Nephrol. 2008;23:787-96.

9. Litwin M, Trelewicz J, Wawer ZT, et al. Intima-media thickness and functional properties of arterial wall in elastic and muscular type arteries in children and adolescents with arterial hypertension: controlled study. Pediatr Nephrol. 2004;19:767-74.

10. • Markus MRP, Stritzke J, Siewert U, et al. Variation in body composition determines long-term blood pressure changes in prehypertension. J Am Coll Cardiol. 2010;56:65-76. Prospective study showing that maintenance of healthy body weight and fitness is associated with maintenance of normal blood pressure over time in older adults.

11. Tirosh A, Afek A, Rudich A, et al. Progression of normotensive adolescent into hypertensive adults. Hypertension. 2010;56:203-9. 
12. • Lever AF, Harrap SB. Essential hypertension: a disorder of growth with origins in childhood ? J Hypertens. 1992;10:101-20. Presentation of hypothesis linking tempo of development and growth spurt with metabolic abnormalities and elevation of blood pressure.

13. Hamilton M, Pickering GW, Roberts JAF, Sowry GSC. The aetiology of essential hypertension I. The arterial pressure in the general population. Clin Sci. 1954;13:273-304.

14. - Katz SH, Hediger ML, Shall JI, et al. Blood pressure, growth, maturation from childhood through adolescence: mixed longitudinal analyses from Philadelphia Blood Pressure Project. Hypertension. 1980;2 Suppl 1:55-69. This study describes relationship between acceleration of biological maturation with elevated blood pressure in adolescents.

15. Pludowski P, Litwin M, Niemirska A, et al. Accelerated skeletal maturation in children with primary hypertension. Hypertension. 2009;54:1234-9. This study describes differences in biological maturation expressed as difference between bone age and chronological age in relation to blood pressure status.

16. Kułaga Z, Litwin M, Tkaczyk M, et al. Polish 2010 growth references for school aged children and adolescents. Eur J Pediatr. 2011;170:599-609.

17. - Halldorsson T, Gunnarsdottir I, Birgisdottir BE, et al. Childhood growth and adult hypertension in a population of high birth weight. Hypertension. 2010;58:8-15. Retrospective study confirming relationship between acceleration of biological development and later development of arterial hypertension in males.

18. - Kivimaki M, Lawlor DA, Smith DG, et al. Association of the age of menarche with cardiovascular risk factors, vascular structure and function in adulthood: the cardiovascular risk in young Finns study. Am J Clin Nutr. 2008;87:1876-82. This study describes relationship between acceleration of biological development with risk of metabolic syndrome and arterial hypertension in young adulthood in women.

19. Litwin M, Śladowska J, Antoniewicz J, et al. Metabolic abnormalities, insulin resistance and metabolic syndrome in children with primary hypertension. Am J Hypertens. 2007;20:875-82.

20. - Feig DI, Johnson RJ. Hyperuricemia in childhood primary hypertension. Hypertension. 2003;42:247-52. This study describes elevation of serum uric acid concentrations as most typical metabolic abnormality in children with primary hypertension.

21. Feig DI, Soletsky B, Johnosn RJ. Effect of allopurinol on blood pressure of adolescent with newly diagnosed essential hypertension: a randomized trial. JAMA. 2008;300:924-32.

22. - Soletsky B, Feig DI. Uric acid reduction rectifies prehypertension in obese adolescents. Hypertension. 2012;60:1148-56. These two studies are the first pediatric studies reporting effects of allopurinol and probenecid on decrease of blood pressure in hypertensive [21] and pregypertensive [22] adolescents.

23. Turi S, Friedman A, Bereczki C, et al. Oxidative stress in juvenile hypertension. J Hypertens. 2003;21:145-52.

24. - Sladowska-Kozlowska J, Litwin M, Niemirska A, et al. Oxidative stress in hypertensive children before and after 1 year of antihypertensive treatment. Pediatr Nephrol. 2012;27:1943-51. It is the first prospective study reporting results of antihypertensive treatment on oxidative stress in children with primary hypertension and relation between OSX and target organ damage.

25. Chae CU, Lee RT, Rifai N, Ridker PM. Blood pressure and inflammation in apparently healthy man. Hypertension. 2001;38:399-403.

26. Festa A, Agostino Jr, Howard G, et al. Chronic subclinical inflammation as a part of the insulin resistance syndrome: the Insulin Resistance Atherosclerosis Study (IRAS). Circulation. 2000;102:42-7.

27. Barzilay JI, Abraham L, Heckbert SR, et al. The relation of markers of inflammation to the development of glucose disorders in the elderly. The Cardiovascular Health Study. Diabetes. 2001;50:2384-9.

28. Fernandez-Real JM, Ricard W. Insulin resistance and chronic cardiovascular inflammatory syndrome. Endocr Rev. 2003;24:278-301.
29. Litwin M, Michalkiewicz J, Niemirska A, et al. Inflammatory activation in children with primary hypertension. Pediatr Nephrol. 2010;25:2489-99.

30. Harrison DG, Vinch A, Lob, Mahdur MS. Role of adaptive immune system in hypertension. Curr Opin Pharmacol. 2010;10:203-7.

31. •• Harrison DG, Guzik TJ, Lob H, et al. Inflammation, immunity and hypertension. Hypertension. 2011;57:132-40. This review describes most important immunological phenomena found in experimental studies and presents hypothesis explaining the role of immune system in pathogenesis of arterial hypertension.

32. Jurewicz M, McDermott DH, Sechler JM, et al. Human T and natural killer cells possess a functional renin-angiotensin system: further mechanisms of angiotensin induced inflammation. J Am Soc Nephrol. 2007;18:1093-102.

33. Hoch NE, Guzik TJ, Chen W, et al. Regulation of T cell function by endogenously produced angiotensin II. Am J Physiol Regul Integr Comp Physiol. 2009;296:R208-16.

34. Benicky J, Sanchez-Lemus E, Pavel J, Saavedra JM. Antiinflammatory effects of angiotensin receptor blockers in the brain and the periphery. Cell Moll Neurobiol. 2009;29:781-92.

35. Chon H, Gaillard CAJM, van der Meijden BB, et al. Broadly altered gene expression in blood leukocytes in essential hypertension is absent during treatment. Hypertension. 2004;43:947-51.

36. Coppo M, Bandinelli M, Berni A et al. Ang-II upregulation of T lymphocyte renin-angiotensin system is amplified by low-grade inflammation in human hypertension. Am J Hypertens. 2011;24: 716-723.

37. Timofeeva AW, Goryunova LE, Khaspekov GL et al. Altered gene expression pattern in peripheral blood leucocytes from patients with arterial hypertension. Ann NY Acad Sci. 2006;1091:319-335.

38. Litwin M, Michałkiewicz, Trojanek J, et al. Altered genes profile of renin-angiotensin system, immune system, and adipokines receptors in leukocytes of children with primary hypertension. Hypertension. 2013;61:431-6.

39. Marvar PJ, Thabet SR, Guzik TJ, et al. Central and peripheral mechanisms of T-lymphocyte activation and vascular inflammation produced by angiotensin II -induced hypertension. Circ Res. 2010;107:263-70.

40. • Guzik TJ, Hoch NE, Brown KA, et al. Role of T cells in the genesis of angiotensin II induced hypertension and vascular dysfunction. J Exp Med. 2007;204:2449-60. In this experimental study role of $T$ cells in pathogenesis of angiotensin-dependent hypertension has been studied.

41. Marvar PJ, Vinch A, Thabet S, et al. T lymphocytes and vascular inflammation contribute to stress-dependent hypertension. Biol Psychiatry. 2012;71:774-82.

42. • Barhoumi T, Kasal DA, Li MW, et al. T regulatory lymphocytes prevent angiotensin II induced hypertension and vascular injury. Hypertension. 2011;57:469-76. A description of the role of T-regs in pathogenesis of arterial injury and elevation of blood pressure.

43. Zhang $\mathrm{W}$, Wang $\mathrm{W}, \mathrm{Yu} \mathrm{H}$, et al. Interleukin 6 underlies angiotensin II induced hypertension and chronic renal damage. Hypertension. 2012;59:136-44.

44. Coppo M, Boddi M, Bandinelli M, et al. Angiotensin II up-regulates renin-angiotensin system in human isolated $\mathrm{T}$ lymphocytes. Regul Pept. 2008;151:1-6.

45. - Marchesi C, Dentali F, Nicolini E, et al. Plasma levels of matrix metalloproteinases and their inhibitors in hypertension: a systematic review and metaanalysis. J Hypertens. 2012;30:3-16. A detailed metaanalysis of studies describing role of MMPs and TIMPs in arterial hypertension.

46. Cheng M, Hashmi S, Mao X, Zeng QT. Relationship of adiponectin matrix metalloproteinase-9 to tissue inhibitor of metalloproteinase-1 ratio with coronary plaque morphology in patients with acute coronary syndrome. Can J Cardiol. 2008;24:385-90.

47. Głowińska-Olszewska B, Urban M. Elevated matrix metallloproteinase 9 and tissue inhibitor of metalloproteinase 1 in obese children and adoelscents. Metabolism. 2007;56:799-805. 
48. Niemirska A, Trojanek J, Michałkiewicz J, Litwin M. Altered matrix metalloproteinases 9 and tissue inhibitor of metalloproteinases 1 activities in children with primary hypertension. 23rd European Meeting on Hypertension and Cardiovascular Protection 2013 (abstract).

49. Stabouli S, Kotsis V, Rizos Z, et al. Left ventricular mass in normotensive, prehypertensive and hypertensive children and adolescents. Pediatr Nephrol. 2009;24:1545-51.

50. Sorof JM, Cardwell G, Franco K, Portman RJ. Ambulatory blood pressure and left ventricular mass in hypertensive children. Hypertension. 2002;39:903-8.

51. Litwin M, Niemirska A, Ruzicka M, Feber J. White coat hypertension in children: not rare and not benign? J Am Soc Hypertens. 2009;3:416-23.

52. Litwin M, Niemirska A, Sladowska J, et al. Left ventricular hypertrophy and arterial wall thickening in children with essential hypertension. Pediatr Nephrol. 2006;21:811-9.

53. - Litwin M, Niemirska, Sladowska-Kozłowska J, et al. Regression of target organ damage in children and adolescents with primary hypertension. Pediatr Nephrol. 2010;25:2489-99. One of the few pediatric prospective studies evaluating effects of antihypertesive treatment on target organ damage and metabolic abnormalities in children with primary hypertension.

54. Sladowska-Kozlowska J, Litwin M, Niemirska A, et al. Change in left ventricular geometry during antihypertensive treatment in children with primary hypertension. Pediatr Nephrol. 2011;26:2201-9.

55. Richey PA, Disessa TG, Hastings MC, et al. Ambulatory blood pressure and increased left ventricular mass in children at risk for hypertension. J Pediatr. 2008;152:343-8.

56. National High Blood Pressure Education Program Working Group on High Blood Pressure in Children and Adolescents. The Fourth Report on Diagnosis, Evaluation and Treatment of High Blood Pressure in Children and Adolescents. Pediatrics. 2004;114:555-76.

57. - Lurbe E, Cifkova R, Cruickshank JK, et al. European Society of Hypertension. Management of high blood pressure in children and adolescents: recommendations of the European Society of Hypertension. J Hypertens. 2009;27:1719-42. The first European Society of Hypertension guidelines concerning treatment of arterial hypertension in children and discussing the significance of metabolic abnormalities in primary hypertension.

58. Schaefer F, Litwin M, Zachwieja J, et al. Efficacy and safety of valsartan compared to enalapril in hypertensive children: a 12week, randomized, double-blind, parallel-group study. J Hypertens. 2011;29:2484-90.

59. Schaefer F, van de Walle J, Zurowska A, et al. Efficacy, safety and pharmacokinetics of candesartan cilexetil in hypertensive children from 1 to less than 6 years of age. J Hypertens. 2010;28:1083-90.

60. Niemirska A, Litwin M, Feber J. Changes in mean arterial pressure/heart rate rhythmicity, target organ damage and visceral obesity in hypertrensive boys on antihypertensive therapy. Pediatr Nephrol. 2012;27:1761. abstract.

61. - O'Rourke MF, Adji A. Guidelines on guidelines: focus on isolated hypertension in youth. J Hypertens. 2013;31:649-54. Important discussion of hemodynamic phenomena of increased brachial blood pressure despite normal central blood pressure in young men.

62. •• Johnson RJ, Rodriguez-Iturbe B, Nakagawa T, Kang DH, Feig DI, Herrera-Acosta J. Subtle renal injury is likely a common mechanism for salt-sensitive essential hypertension. Hypertension. 2005;45:326-30. Presentation of hypothesis linking primary activation of sympathetic nervous system with arteriolar constriction, metabolic abnormalities and evolution to sodium dependent hypertension.

63. - Zubcevic J, Waki H, Raizada MK, Paton JFR. Autonomicimmune-vascular interaction: an emerging concept for neurogenic hypertension. Hypertension. 2011;57:1026-33. Review of experimental studies showing interactions between immune vascular injury and activation of autonomic centers in central nervous system. 